PROCEEDINGS OF THE

AMERICAN MATHEMATICAL SOCIETY

Volume 140, Number 1, January 2012, Pages 325-335

S 0002-9939(2011)11286-3

Article electronically published on August 11, 2011

\title{
A METRIC BETWEEN QUASI-ISOMETRIC TREES
}

\author{
ÁLVARO MARTÍNEZ-PÉREZ \\ (Communicated by Alexander N. Dranishnikov)
}

\begin{abstract}
It is known that PQ-symmetric maps on the boundary characterize the quasi-isometry type of visual hyperbolic spaces, in particular, of geodesically complete $\mathbb{R}$-trees. We define a map on pairs of PQ-symmetric ultrametric spaces which characterizes the branching of the space. We also show that when the ultrametric spaces are the corresponding end spaces, this map defines a metric between rooted geodesically complete simplicial trees with minimal vertex degree 3 in the same quasi-isometry class. Moreover, this metric measures how far the trees are from being rooted isometric.
\end{abstract}

\section{INTRODUCTION}

The study of quasi-isometries between trees and the induced maps on their end spaces has a voluminous literature. This is often set in the more general context of hyperbolic metric spaces and their boundaries. See Bonk and Schramm [2], Buyalo and Schroeder [4, Ghys and de la Harpe [6], and Paulin [14] to name a few.

For a quasi-isometry $f: X \rightarrow Y$ between Gromov hyperbolic, almost geodesic metric spaces, Bonk and Schramm define 2, Proposition 6.3] the induced map $\partial f: \partial X \rightarrow \partial Y$ between the boundaries at infinity and prove [2, Theorem 6.5] that $\partial f$ is $\mathrm{PQ}$-symmetric with respect to any metrics on $\partial X$ and $\partial Y$ in their canonical gauges. Moreover, they prove that a PQ-symmetric map between bounded metric spaces can be extended to a map between their hyperbolic cones and obtain that a PQ-symmetric map between the boundaries at infinity of Gromov hyperbolic, almost geodesic metric spaces, implies a quasi-isometry equivalence between the spaces. In the particular case that $X$ and $Y$ are $\mathbb{R}$-trees, then $\partial X=e n d(X, v)$, $\partial Y=\operatorname{end}(Y, w)$ and the end space metrics are in the canonical gauges for any choice of roots.

Another source for the result that quasi-isometries between $\mathbb{R}$-trees induce PQsymmetric homeomorphisms on their ultrametric end spaces is [4, Theorem 5.2.17] from Buyalo and Schroeder. They work with Gromov hyperbolic, geodesic metric spaces and with visual boundaries on their boundaries. When specialized to $\mathbb{R}$ trees, these boundaries are the ultrametric end spaces. For the converse of this approach, see [12].

Received by the editors August 6, 2010.

2010 Mathematics Subject Classification. Primary 54E40, 30C65, 53C23; Secondary 54E40.

Key words and phrases. Tree, real tree, ultrametric, end space, bounded distortion equivalence, quasi-isometry, PQ-symmetric, pseudo-doubling metric space.

The author was partially supported by MTM 2009-07030.

(C)2011 American Mathematical Society Reverts to public domain 28 years from publication 
In [10] we defined bounded distortion equivalences and we proved that bounded distortion equivalences characterize PQ-symmetric homeomorphisms between certain classes of bounded, complete, uniformly perfect, ultrametric spaces which we called pseudo-doubling. This class includes those ultrametric spaces arising up to similarity as the end spaces of bushy simplicial trees.

Bounded distortion equivalences can be seen from a geometrical point of view as a coarse version of quasiconformal homeomorphisms (see for example [1, 7] and 8. for a geometric approach to quasiconformal maps) where instead of looking at the distortion of the spheres with the radius tending to 0 we consider the distortion of all of them.

Of central importance in the theory of quasiconformal mappings are Teichmüller spaces. The Teichmüller space is the set of Riemannian surfaces of a given quasiconformal type, and the Teichmüller metric measures how far are the spaces from being conformally equivalent. There is also an extensive literature on this; see for example [1] and [8]. The question we deal with in this paper is to see if something similar to a Teichmüller metric can be defined with these bounded distortion equivalences playing the role of the quasiconformal homeomorphisms.

Here we consider the set of ultrametric spaces of a given PQ-symmetry type. What is obtained is a function $\varrho$ (see section 3) which is not a metric in the general framework of pseudo-doubling ultrametric spaces because it fails to hold the triangle inequality. Nevertheless, adding a condition on the metrics, it is enough to characterize what we call here the branching of the space, which is a natural concept when looking at the ultrametric space as the boundary of a tree.

Theorem 1.1. Let $(U, d),\left(U^{\prime}, d^{\prime}\right)$ be ultrametric spaces. If the metrics $d, d^{\prime}$ are pseudo-discrete, then $\varrho\left(U, U^{\prime}\right)=0$ if and only if $(U, d)$ and $\left(U^{\prime}, d^{\prime}\right)$ have the same branching.

As we mentioned before, if the ultrametric spaces are end spaces of $\mathbb{R}$-trees, then the PQ-symmetry type of the boundary corresponds to the quasi-isometry type of the trees. In the case of a quasi-isometry class, $[(R, z)]$, of rooted geodesically complete simplicial trees with minimal vertex degree 3 , we prove

Theorem 1.2. The function $\varrho$ is an unbounded metric on $[(R, z)]$.

This means that in the category of rooted simplicial trees with minimal vertex degree 3, the branching is enough to characterize the isometry type, the quasiisometry type and the "distance" between quasi-isometric objects.

\section{Preliminaries on trees, end spaces, and ultrametrics}

In this section, we recall the definitions on trees and their end spaces that are relevant to this paper. We also describe a well-known correspondence between trees and ultrametric spaces. See Fernberg [5] for an early result along these lines and Hughes 9 for additional background.

Definition 2.1. Let $(T, d)$ be a metric space.

(1) $(T, d)$ is an $\mathbb{R}$-tree if $T$ is uniquely arcwise connected and for all $x, y \in T$, the unique arc from $x$ to $y$, denoted $[x, y]$, is isometric to the subinterval $[0, d(x, y)]$ of $\mathbb{R}$.

(2) A rooted $\mathbb{R}$-tree $(T, v)$ consists of an $\mathbb{R}$-tree $(T, d)$ and a point $v \in T$, called the root. 
(3) A rooted $\mathbb{R}$-tree $(T, v)$ is geodesically complete if every isometric embedding $f:[0, t] \rightarrow T$ with $t>0$ and $f(0)=v$ extends to an isometric embedding $F:[0, \infty) \rightarrow T$. This is often also called "with extendable geodesics".

(4) A simplicial $\mathbb{R}$-tree is an $\mathbb{R}$-tree $(T, d)$ such that $T$ is the (geometric realization of) a simplicial complex and every edge of $T$ is isometric to the closed unit interval $[0,1]$.

Notation 2.2. For any $x \in(T, v)$, let $\|x\|=d(x, v)$.

Definition 2.3. An ultrametric space is a metric space $(X, d)$ such that $d(x, y) \leq$ $\max \{d(x, z), d(z, y)\}$ for all $x, y, z \in X$.

Definition 2.4. The end space of a rooted $\mathbb{R}$-tree $(T, v)$ is given by:

$\operatorname{end}(T, v)=\{F:[0, \infty) \rightarrow T \mid F(0)=v$ and $F$ is an isometric embedding $\}$.

Let $F, G \in \operatorname{end}(T, v)$.

(1) The Gromov product at infinity is $(F \mid G)_{v}:=\sup \{t \geq 0 \mid F(t)=G(t)\}$.

(2) The end space metric is $d_{v}(F, G):=\mathrm{e}^{-(F \mid G)_{v}}$.

(3) The arc $F\left(\left[0,(F \mid G)_{v}\right]\right)$ is denoted by $[F \mid G]$.

Proposition 2.5. If $(T, v)$ is a rooted $\mathbb{R}$-tree, then $\left(\operatorname{end}(T, v), d_{v}\right)$ is a complete ultrametric space of diameter $\leq 1$.

In this article a map is a function that need not be continuous.

Definition 2.6. A map $f: X \rightarrow Y$ between metric spaces $\left(X, d_{X}\right)$ and $\left(Y, d_{Y}\right)$ is a quasi-isometric map if there are constants $\lambda \geq 1$ and $A>0$ such that for all $x, x^{\prime} \in X$,

$$
\frac{1}{\lambda} d_{X}\left(x, x^{\prime}\right)-A \leq d_{Y}\left(f(x), f\left(x^{\prime}\right)\right) \leq \lambda d_{X}\left(x, x^{\prime}\right)+A .
$$

If $f(X)$ is a net in $Y$ (i.e., there exists $\epsilon>0$ such that for each $y \in Y$ there exists $x \in X$ such that $\left.d_{Y}(f(x), y)<\epsilon\right)$, then $f$ is a quasi-isometry and $X, Y$ are quasi-isometric.

Definition 2.7. A map $f: X \rightarrow Y$ between metric spaces is called quasi-symmetric if it is not constant and if there is a homeomorphism $\eta:[0, \infty) \rightarrow[0, \infty)$ such that from $|x a| \leq t|x b|$ it follows that $|f(x) f(a)| \leq \eta(t)|f(x) f(b)|$ for any $a, b, x \in X$ and all $t \geq 0$. The function $\eta$ is called the control function of $f$.

Definition 2.8. A quasi-symmetric map is said to be power quasi-symmetric or $P Q$-symmetric if its control function is of the form

$$
\eta(t)=q \max \left\{t^{p}, t^{\frac{1}{p}}\right\}
$$

for some $p, q \geq 1$.

\section{Bounded Distortion EQUiVAlENCES BETWEEN ULTRAMETRIC SPACES}

Let us recall some definitions as stated in [10].

Definition 3.1. Let $f: X \rightarrow Y$ be a homeomorphism between metric spaces $\left(X, d_{X}\right)$ and $\left(Y, d_{Y}\right)$. If $x_{0} \in X$ and $\varepsilon>0$, then the distortion by $f$ of the $\varepsilon$ sphere $S\left(x_{0}, \varepsilon\right):=\left\{x \in X \mid d_{X}\left(x_{0}, x\right)=\varepsilon\right\}$ at $x_{0}$ is

$$
D_{f}\left(x_{0}, \varepsilon\right):=\left\{\begin{array}{cc}
\frac{\sup \left\{d_{Y}\left(f\left(x_{0}\right), f(x)\right) \mid d_{X}\left(x_{0}, x\right)=\varepsilon\right\}}{\inf \left\{d_{Y}\left(f\left(x_{0}\right), f(x)\right) \mid d_{X}\left(x_{0}, x\right)=\varepsilon\right\}} & \text { if } S\left(x_{0}, \varepsilon\right) \neq \emptyset \\
1 & \text { if } S\left(x_{0}, \varepsilon\right)=\emptyset .
\end{array}\right.
$$


Definition 3.2. Let $f: X \rightarrow Y$ be a homeomorphism between metric spaces.

(1) $f$ is conformal if $\limsup _{\varepsilon \rightarrow 0} D_{f}\left(x_{0}, \varepsilon\right)=1$ for all $x_{0} \in X$.

(2) $f$ is $K$-quasiconformal, where $K>0$, if $\limsup _{\varepsilon \rightarrow 0} D_{f}(x, \varepsilon) \leq K$ for all $x \in X$.

(3) $f$ is quasiconformal if $f$ is $K$-quasiconformal for some $K>0$.

(4) $f$ has bounded distortion if there exists $K>0$ such that

$$
\sup _{x \in X} \sup _{\varepsilon>0} D_{f}(x, \varepsilon) \leq K .
$$

(5) $f$ is a bounded distortion equivalence if $f$ and $f^{-1}: Y \rightarrow X$ have bounded distortion.

Consider $(U, d),\left(U^{\prime}, d^{\prime}\right)$ to be two bounded distortion equivalent ultrametric spaces. Let $\mathcal{K}_{U, U^{\prime}}$ be the greatest lower bound for $K$ such that there exists a homeomorphism $f: U \rightarrow U^{\prime}$ with $\sup _{x \in U} \sup _{\varepsilon>0} D_{f}(x, \varepsilon) \leq K$ and $\sup _{x^{\prime} \in U^{\prime}} \sup _{\varepsilon>0} D_{f^{-1}}\left(x^{\prime}, \varepsilon\right) \leq$ $K$. Then, given $\mathcal{U}$ a bounded distortion equivalence class of ultrametric spaces, let us define $\varrho: \mathcal{U} \times \mathcal{U} \rightarrow \mathbb{R}_{+}$such that

$$
\varrho\left(U, U^{\prime}\right):=\ln \left(1+2 \ln \left(\mathcal{K}_{U, U^{\prime}}\right)\right) .
$$

Definition 3.3. Given a bijection $h:(U, d) \rightarrow\left(U^{\prime}, d^{\prime}\right)$ between ultrametric spaces, we say that $h$ preserves the branching if given three points $x, y, z \in U, d(x, y)=$ $d(x, z)$ implies that $d^{\prime}(h(x), h(y))=d^{\prime}(h(x), h(z))$ and $d(x, y)<d(x, z)$ implies that $d^{\prime}(h(x), h(y))<d^{\prime}(h(x), h(z))$ (i.e. $d(x, y)=d(x, z)$ if and only if $d^{\prime}(h(x), h(y))=$ $\left.d^{\prime}(h(x), h(z))\right)$. If there exists such a bijection, we say that the ultrametric spaces have the same branching.

Remark 3.4. Note that this defines an equivalence relation.

Definition 3.5. Let $(X, d)$ be a metric space. We say that $d$ is pseudo-discrete if there is some $\delta>1$ such that for every nonempty sphere $S(x, r)$ and any $y$ such that $\frac{r}{\delta}<d(y, x)<r \cdot \delta, d(x, y)=r$.

Theorem 3.6. Let $(U, d),\left(U^{\prime}, d^{\prime}\right)$ be ultrametric spaces. If the metrics $d, d^{\prime}$ are pseudo-discrete, then $\varrho\left(U, U^{\prime}\right)=0$ if and only if $(U, d)$ and $\left(U^{\prime}, d^{\prime}\right)$ have the same branching.

Proof. If the branching is the same, then there is a homeomorphism $h$ such that the spheres are preserved; that is, $D_{h}(x, r)=1$ and $D_{h^{-1}(h(x), r)}=1$ for any $x \in U$ and any $r>0$.

Suppose that both ultrametric spaces are pseudo-discrete with the same constant $\delta>1$ and let $\varrho\left(U, U^{\prime}\right)=0$, which is equivalent to saying that $\mathcal{K}_{U, U^{\prime}}=1$. Then, for any $\varepsilon>1$ there exists a homeomorphism $h_{\varepsilon}: U \rightarrow U^{\prime}$ such that given $x, y, z \in U$ with $d(x, y)=d(x, z)$, then, assuming $d^{\prime}\left(h_{\varepsilon}(x), h_{\varepsilon}(y)\right) \leq d^{\prime}\left(h_{\varepsilon}(x), h_{\varepsilon}(z)\right)_{w}$,

$$
\frac{d^{\prime}\left(h_{\varepsilon}(x), h_{\varepsilon}(z)\right)}{d^{\prime}\left(h_{\varepsilon}(x), h_{\varepsilon}(y)\right)}<\varepsilon .
$$

Taking $\varepsilon<\delta$, we conclude that $d^{\prime}\left(h_{\varepsilon}(x), h_{\varepsilon}(y)\right)=d^{\prime}\left(h_{\varepsilon}(x), h_{\varepsilon}(z)\right)$.

Now suppose that $d(x, y)<d(x, z)$. Then $d(y, z)=d(x, z)$ by the properties of the ultrametric. Hence, as we just proved, taking $\epsilon<\delta, d^{\prime}\left(h_{\varepsilon}(y), h_{\varepsilon}(z)\right)=$ $d^{\prime}\left(h_{\varepsilon}(x), h_{\varepsilon}(z)\right)$ and therefore $d^{\prime}\left(h_{\varepsilon}(x), h_{\varepsilon}(y)\right) \leq d^{\prime}\left(h_{\varepsilon}(x), h_{\varepsilon}(z)\right)$.

If $d^{\prime}\left(h_{\varepsilon}(x), h_{\varepsilon}(z)\right)=d^{\prime}\left(h_{\varepsilon}(x), h_{\varepsilon}(y)\right)$ the same argument on $h^{-1}$ implies that $d(x, y)=d(x, z)$, leading to a contradiction. Thus, $d^{\prime}\left(h_{\varepsilon}(x), h_{\varepsilon}(y)\right)<d^{\prime}\left(h_{\varepsilon}(x)\right.$, $\left.h_{\varepsilon}(y)\right)$, finishing the proof. 
Let us recall the following definition from [10.

Definition 3.7. A metric space is pseudo-doubling if for every $C>1$ there exist $N \in \mathbb{N}$ such that: if $0<r<R$ with $R / r=C$ and $x \in X$, then there are at most $N$ balls $B$ such that $B(x, r) \subseteq B \subseteq B(x, R)$.

Remark 3.8. It is immediate to check that if $(X, d)$ is a metric space and $d$ is pseudo-discrete, then $(X, d)$ is pseudo-doubling.

Nevertheless, two pseudo-doubling ultrametric spaces $U, U^{\prime}$ with $\varrho\left(U, U^{\prime}\right)=0$ need not have the same branching as shown in Example 3.12

Consider $(T, v),\left(T^{\prime}, w\right)$ to be rooted geodesically complete $\mathbb{R}$-trees such that $\operatorname{end}(T, v)$ and $\operatorname{end}\left(T^{\prime}, w\right)$ are bounded distortion equivalent. When we are considering rooted trees we will denote $\mathcal{K}_{e n d(T, v), e n d\left(T^{\prime}, w\right)}$ just by $\mathcal{K}_{(T, v),\left(T^{\prime}, w\right)}$, and, therefore, we can define

$$
\varrho\left((T, v),\left(T^{\prime}, w\right)\right):=\ln \left(1+2 \ln \left(\mathcal{K}_{(T, v),\left(T^{\prime}, w\right)}\right)\right) .
$$

Now, translating Definition 3.3 to trees:

Remark 3.9. Let $(T, v),\left(T^{\prime}, w\right)$ be rooted geodesically complete $\mathbb{R}$-trees. A bijection $h: \operatorname{end}(T, v) \rightarrow \operatorname{end}\left(T^{\prime}, w\right)$ preserves the branching if for any $F, G, H \in \operatorname{end}(T, v)$, if $(F \mid G)_{v}=(F \mid H)_{v}$, then $(h(F) \mid h(G))_{v}=(h(F) \mid h(H))_{v}$ and if $(F \mid G)_{v}<(F \mid H)_{v}$, then $(h(F) \mid h(G))_{v}<(h(F) \mid h(H))_{v}$. If there exists such a bijection, we say that $(T, v)$ and $\left(T^{\prime}, w\right)$ have the same branching.

Now, from Proposition 3.6, we have:

Corollary 3.10. Let $(T, v),\left(T^{\prime}, w\right)$ be rooted geodesically complete $\mathbb{R}$-trees. If the metrics $d_{v}, d_{w}$ of end $(T, v), \operatorname{end}(T, w)$ are pseudo-discrete, then $\varrho\left((T, v),\left(T^{\prime}, w\right)\right)=$ 0 if and only if the ramifications of $(T, v)$ and $\left(T^{\prime}, w\right)$ are the same.

In particular, the end space metric of a rooted geodesically complete simplicial tree is pseudo-discrete with $\delta=e$.

Corollary 3.11. If $(T, v),\left(T^{\prime}, w\right)$ are rooted simplicial geodesically complete $\mathbb{R}-$ trees, then $\varrho\left((T, v),\left(T^{\prime}, w\right)\right)=0$ if and only if the ramifications of $(T, v)$ and $\left(T^{\prime}, w\right)$ are the same.

This is not true in general for rooted geodesically complete $\mathbb{R}$-trees as we can see in Example 3.12 .

Example 3.12. Let $(T, v),\left(T^{\prime}, w\right)$ be two rooted geodesically complete $\mathbb{R}$-trees such that $\operatorname{end}(T, v)=\left\{F_{n}, G_{n}, H_{n} \mid n=0,1,2, \ldots\right\}$ and $\operatorname{end}\left(T^{\prime}, w\right)=\left\{F_{n}^{\prime}, G_{n}^{\prime}, H_{n}^{\prime} \mid\right.$ $n=1,2, \ldots\}$ and the following relations hold (see Figure 1).

For $\operatorname{end}(T, v)$ :

- $\left(F_{i} \mid F_{j}\right)_{v}=0 \forall i \neq j$.

- $\left(F_{0} \mid G_{0}\right)_{v}=\left(F_{0} \mid H_{0}\right)_{v}=1$.

- $\left(F_{i} \mid G_{i}\right)_{v}=1 \forall i \geq 1$.

- $\left(F_{i} \mid H_{i}\right)_{v}=2 \forall i \geq 1$.

For $\operatorname{end}\left(T^{\prime}, w\right)$ :

- $\left(F_{i}^{\prime} \mid F_{j}^{\prime}\right)_{w}=0 \forall i \neq j$.

- $\left(F_{i}^{\prime} \mid G_{i}^{\prime}\right)_{v}=1 \forall i \geq 1$.

- $\left(F_{i}^{\prime} \mid H_{i}^{\prime}\right)_{v}=1+\frac{1}{i} \forall i \geq 1$. 

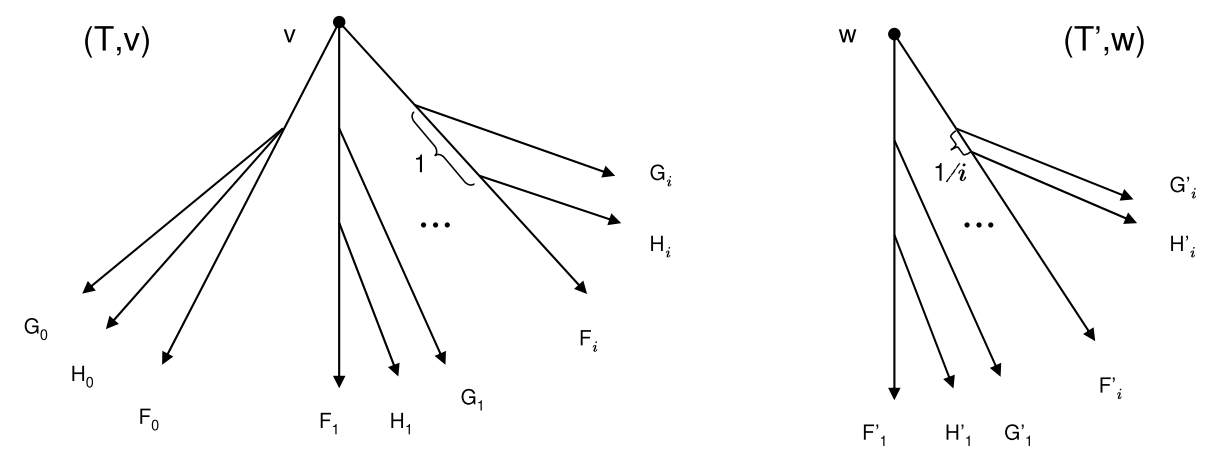

Figure 1. $\varrho\left((T, v),\left(T^{\prime}, w\right)\right)=0$ with the trees having pseudodoubling end spaces and different branching.

Let us define $h_{n}: \operatorname{end}(T, v) \rightarrow \operatorname{end}\left(T^{\prime}, w\right)$ such that $h_{n}\left(F_{0}\right)=F_{n}^{\prime}, h_{n}\left(G_{0}\right)=G_{n}^{\prime}$, $h_{n}\left(H_{0}\right)=H_{n}^{\prime}, h_{n}\left(F_{i}\right)=F_{i}^{\prime}, h_{n}\left(G_{i}\right)=G_{i}^{\prime}, h_{n}\left(H_{i}\right)=H_{i}^{\prime} \forall 1 \leq i<n$ and $h_{n}\left(F_{i}\right)=$ $F_{i+1}^{\prime}, h_{n}\left(G_{i}\right)=G_{i+1}^{\prime}$ and $h_{n}\left(H_{i}\right)=H_{i+1}^{\prime} \forall i \geq n$.

It is immediate to check that there is no sphere distorted by $h_{n}^{-1}$ and the unique spheres distorted by $h_{n}$ are $S_{1}\left(F_{0}\right)$ and $S_{1}\left(H_{0}\right)$ in end $(T, v)$, where $D_{h_{n}}\left(F_{0}, 1\right)=$ $e^{\frac{1}{n}}=D_{h_{n}}\left(G_{0}, 1\right)$. Therefore, for any $K>1$, there exist some $n \in \mathbb{N}$ such that $e^{\frac{1}{n}}<K$ and $\mathcal{K}_{(T, v),\left(T^{\prime}, w\right)}=1$, which implies that $\varrho\left((T, v),\left(T^{\prime}, w\right)\right)=0$.

Clearly, the ramification is not the same. From the cardinality together with the bounded distortion condition we know that $h\left(S_{1}\left(F_{i}\right)\right)$ corresponds to $S_{1}\left(h\left(F_{i}^{\prime}\right)\right)$ for any $i$, and in $(T, v)$ we have $\left(F_{0} \mid G_{0}\right)_{v}=\left(F_{0} \mid H_{0}\right)_{v}=\left(G_{0} \mid H_{0}\right)_{v}$ while for any bijection $h: \operatorname{end}(T, v) \rightarrow \operatorname{end}\left(T^{\prime}, w\right)$ either $\left(h\left(F_{0}\right) \mid h\left(G_{0}\right)\right)_{w} \neq\left(h\left(F_{0}\right) \mid h\left(H_{0}\right)\right)_{w}$ or $\left(h\left(F_{0}\right) \mid h\left(G_{0}\right)\right)_{w} \neq\left(h\left(G_{0}\right) \mid h\left(H_{0}\right)\right)_{w}$.

\section{A metric Between QUasi-Isometric trees}

Definition 4.1. An $\mathbb{R}$-tree $T$ is bushy if there is a constant $K>0$, called a bushy constant, such that for any point $x \in T$ there is a point $y \in T$ such that $d(x, y)<K$ and $T \backslash\{y\}$ has at least 3 unbounded components.

The following result is a particular case of [10, Theorem 1.1].

Theorem 4.2. A homeomorphism $h: \operatorname{end}(T, v) \rightarrow \operatorname{end}\left(T^{\prime}, w\right)$ between the end spaces of rooted, geodesically complete, simplicial, bushy $\mathbb{R}$-trees is $P Q$-symmetric if and only if $h$ is a bounded distortion equivalence.

A map $f:(T, v) \rightarrow\left(T^{\prime}, w\right)$ is a rooted isometry if it is an isometry and $f(v)=w$. If such a map exists, we say that $(T, v)$ and $\left(T^{\prime}, w\right)$ are rooted isometric, which defines an equivalence relation.

Let us consider, from now on, the category $\mathcal{T}$ of rooted isometry classes of rooted geodesically complete simplicial bushy trees.

Given $(S, x) \in \mathcal{T}$, let $[(S, x)]$ be the class of rooted geodesically complete $\mathbb{R}$-trees quasi-isometric to $(S, x)$ (i.e. whose end space is bounded distortion equivalent to $\operatorname{end}(S, x))$.

Let us recall that, from [2] (see also [12]), as a particular case we have that

Proposition 4.3. end $(T, v)$ and end $\left(T^{\prime}, w\right)$ are $P Q$-symmetric if and only if $(T, v)$ and $\left(T^{\prime}, w\right)$ are quasi-isometric. 
Let us consider the restriction of the category $\mathcal{T}$ to rooted geodesically complete simplicial trees with valence at least 3 at each vertex, $\mathcal{T}_{\geq 3}$. Let $[(R, z)] \subset \mathcal{T}_{\geq 3}$ be the class of trees quasi-isometric to $(R, z)$ (i.e. whose end space is bounded distortion equivalent to $\operatorname{end}(R, z))$.

Proposition 4.4. Given $(T, v),\left(T^{\prime}, w\right) \in[(R, z)], \varrho\left((T, v),\left(T^{\prime}, w\right)\right)=0$ if and only if there is an isometry between end $(T, v)$ and end $\left(T^{\prime}, w\right)$.

Proof. The if part is obvious.

Suppose that $\varrho\left((T, v),\left(T^{\prime}, w\right)\right)=0$. Since the trees are simplicial, $D_{g}(F, r) \in$ $\left\{e^{k} \mid k=0,1, \ldots\right\}$ for any homeomorphism $g$ and any radius $r>0$. Therefore, there is a homeomorphism $h: \operatorname{end}(T, v) \rightarrow \operatorname{end}\left(T^{\prime}, w\right)$ such that $D_{h}(F, r)=1$ and $D_{h^{-1}}\left(F^{\prime}, r\right)=1$ for any $F \in \operatorname{end}(T, v)$, any $F^{\prime} \in \operatorname{end}\left(T^{\prime}, w\right)$ and any $r>0$. Let us prove that $h$ is an isometry by induction on the Gromov product.

Let $F, G \in \operatorname{end}(T, v)$ such that $(F \mid G)_{v}=0$, i.e., $d_{v}(F, G)=1$ and suppose $(h(F) \mid h(G))_{w} \geq 1$. Since the minimal vertex degree is 3 , there is a point $H^{\prime} \in$ $\operatorname{end}\left(T^{\prime}, v\right)$ such that $\left(h(F) \mid H^{\prime}\right)_{w}=0$ and $\left(H^{\prime} \mid h(G)\right)_{w}=0$. Since $D_{h^{-1}}\left(H^{\prime}, 1\right)=1$, necessarily $\left(F \mid h^{-1}\left(H^{\prime}\right)\right)_{v}=0$ and $\left(h^{-1}\left(H^{\prime}\right) \mid G\right)_{v}=0$, but then $D_{h}(F, 1) \geq e$, leading to a contradiction. The same works for $h^{-1}$.

Now suppose that for every $k<n$ and every pair of end points $F_{1}, G_{1} \in \operatorname{end}(T, v)$ (resp. $\left.F_{1}^{\prime}, G_{1}^{\prime} \in \operatorname{end}\left(T^{\prime}, w\right)\right)$ such that $\left(F_{1} \mid G_{1}\right)_{v}=k\left(\operatorname{resp} . \quad\left(F_{1}^{\prime} \mid G_{1}^{\prime}\right)_{w}=k\right)$, then $\left(h\left(F_{1}\right) \mid h\left(G_{1}\right)\right)_{w}=k\left(\operatorname{resp} .\left(h^{-1}\left(F_{1}^{\prime}\right) \mid h^{-1}\left(G_{1}^{\prime}\right)\right)_{w}=k\right)$. Let $F_{2}, G_{2} \in \operatorname{end}(T, v)$ such that $\left(F_{2} \mid G_{2}\right)_{v}=n$ and suppose that $\left(h\left(F_{2}\right) \mid h\left(G_{2}\right)\right)_{w} \neq n$. If $\left(h\left(F_{2}\right) \mid h\left(G_{2}\right)\right)_{w}<n$, then the assumption on $h^{-1}$ implies that $\left(F_{2} \mid G_{2}\right)_{v}<n$. Hence $\left(h\left(F_{2}\right) \mid h\left(G_{2}\right)\right)_{w}>n$, and since $T^{\prime}$ has minimal vertex degree 3 , there exist some $H^{\prime}$ with $\left(h\left(F_{2}\right) \mid H^{\prime}\right)_{w}=$ $n=\left(H^{\prime} \mid h\left(G_{2}\right)\right)_{w}$. Since $D_{h^{-1}}\left(H^{\prime}, e^{-n}\right)=0$ and by the induction hypothesis $\left(F_{2} \mid h^{-1}\left(H^{\prime}\right)\right)_{v},\left(h^{-1}\left(H^{\prime}\right) \mid G_{2}\right)_{v} \geq n$, then $\left(F_{2} \mid h^{-1}\left(H^{\prime}\right)\right)_{v}=n=\left(h^{-1}\left(H^{\prime}\right) \mid G_{2}\right)_{v}$, which leads to a contradiction with $D_{h}\left(F_{2}, e^{-n}\right)=1$.

Proposition 4.5 (9, Corollary 8.7]). Two geodesically complete rooted $\mathbb{R}$-trees, $(T, v)$ and $(S, w)$, are rooted isometric if and only if end $(T, v)$ and end $(S, w)$ are isometric.

From Propositions 4.4 and 4.5 we obtain:

Corollary 4.6. Given $(T, v),\left(T^{\prime}, w\right) \in[(R, z)], \varrho\left((T, v),\left(T^{\prime}, w\right)\right)=0$ if and only if there is a rooted isometry between $(T, v)$ and $\left(T^{\prime}, w\right)$.

Remark 4.7. Note that this distance depends on the ramification of the tree and not on its isometry type. Therefore, the root plays an important role and it is immediate to check that from the same tree with two different roots $(T, v),(T, w)$ it may happen that $\varrho((T, v),(T, w))>0$. Nevertheless, it can be bounded above by a constant depending on the distance between the roots. In fact, $\varrho((T, v),(T, w)) \leq$ $\ln (1+d(v, w))$.

Proposition 4.8. $\varrho$ is a metric in $[(R, z)]$.

Proof. $\varrho$ is real-valued and nonnegative by definition. By Corollary 4.6, $\varrho((T, v)$, $\left.\left(T^{\prime}, w\right)\right)=0$ if and only if $(T, v)=\left(T^{\prime}, w\right)$.

The symmetric property follows immediately from the definition. Hence, it suffices to check the triangle inequality.

Let $(T, v),\left(T^{\prime}, w\right),\left(T^{\prime \prime}, x\right) \in[(R, z)]$ with

$$
\varrho\left((T, v),\left(T^{\prime}, w\right)\right)=d_{1}=\ln \left(1+2 \ln \left(\mathcal{K}_{(T, v),\left(T^{\prime}, w\right)}\right)\right)
$$


and

$$
\varrho\left(\left(T^{\prime}, w\right),\left(T^{\prime \prime}, x\right)\right)=d_{2}=\ln \left(1+2 \ln \left(\mathcal{K}_{\left(T^{\prime}, w\right),\left(T^{\prime \prime}, x\right)}\right)\right) .
$$

To simplify the notation through the proof let us denote $\mathcal{K}_{(T, v),\left(T^{\prime}, w\right)}$ as $\mathcal{K}_{1}$ and $\mathcal{K}_{\left(T^{\prime}, w\right),\left(T^{\prime \prime}, x\right)}$ as $\mathcal{K}_{2}$.

Since $\mathcal{K}_{1}$ and $\mathcal{K}_{2}$ are greatest lower bounds, then there is a homeomorphism $h_{1}: \operatorname{end}(T, v) \rightarrow \operatorname{end}\left(T^{\prime} w\right)$ such that

$$
\sup _{F \in \operatorname{end}(T, v)} \sup _{\varepsilon>0} D_{h_{1}}(F, \varepsilon) \leq \frac{e}{2} \mathcal{K}_{1} \text { and } \sup _{F^{\prime} \in \operatorname{end}\left(T^{\prime}, w\right)} \sup _{\varepsilon>0} D_{h_{1}^{-1}}\left(F^{\prime}, \varepsilon\right) \leq \frac{e}{2} \mathcal{K}_{1},
$$

and a homeomorphism $h_{2}: \operatorname{end}\left(T^{\prime}, w\right) \rightarrow \operatorname{end}\left(T^{\prime \prime}, x\right)$ with the corresponding condition for $\mathcal{K}_{2}$. Also, since the trees are simplicial, for any homeomorphism $h, D_{h}(F, \varepsilon)$ takes values in $\left\{e^{n} \mid n \in \mathbb{N}\right\}$. Hence, $D_{h_{1}}(F, \varepsilon) \leq \mathcal{K}_{1}, D_{h_{1}^{-1}}\left(F^{\prime}, \varepsilon\right) \leq \mathcal{K}_{1}$ and the same happens with $h_{2}$.

Case 1. $\mathcal{K}_{1}=1$. Then $\varrho\left((T, v),\left(T^{\prime \prime}, x\right)\right)=\varrho\left((T, v),\left(T^{\prime}, w\right)\right)+\varrho\left(\left(T^{\prime}, w\right),\left(T^{\prime \prime}, x\right)\right)=$ $\varrho\left(\left(T^{\prime}, w\right),\left(T^{\prime \prime}, x\right)\right)$. It suffices to observe that for any $F \in \operatorname{end}(T, v)$ and $G, H \in$ $S(F, \varepsilon)$, there exists some $\varepsilon^{\prime}>0$ such that $h_{1}(G), h_{1}(H) \in S\left(h_{1}(F), \varepsilon^{\prime}\right)$ and, assuming $d_{x}\left(h_{2}\left(h_{1}(F)\right), h_{2}\left(h_{1}(H)\right)\right) \leq d_{x}\left(h_{2}\left(h_{1}(F)\right), h_{2}\left(h_{1}(G)\right)\right)$, then

$$
\frac{d_{x}\left(h_{2}\left(h_{1}(F)\right), h_{2}\left(h_{1}(G)\right)\right)}{d_{x}\left(h_{2}\left(h_{1}(F)\right), h_{2}\left(h_{1}(H)\right)\right)} \leq \mathcal{K}_{2} .
$$

Case 2. If $\mathcal{K}_{1}>1$, then $\mathcal{K}_{1} \geq e$ and $\ln \mathcal{K}_{1} \geq 1$.

Suppose $F \in \operatorname{end}(T, v)$ and $G, H \in S(F, \varepsilon)$ and let us assume that

$$
t_{0}=\left(h_{1}(G) \mid h_{1}(F)\right)_{w} \leq\left(h_{1}(H) \mid h_{1}(F)\right)_{w}=t_{1} .
$$

Then, since $D_{h_{1}}(F, \varepsilon) \leq \mathcal{K}_{1}$,

$$
t_{1}-t_{0} \leq \ln \left(\mathcal{K}_{1}\right) .
$$

Claim.

$\left|\left(h_{2}\left(h_{1}\right)(F) \mid h_{2}\left(h_{1}(H)\right)\right)_{x}-\left(h_{2}\left(h_{1}\right)(F) \mid h_{2}\left(h_{1}(G)\right)\right)_{x}\right| \leq \ln \left(\mathcal{K}_{1}\right) \cdot \ln \left(\mathcal{K}_{2}\right)+2 \ln \left(\mathcal{K}_{2}\right)+1$.

If $\left(h_{2}\left(h_{1}\right)(F) \mid h_{2}\left(h_{1}(H)\right)\right)_{x}<\left(h_{2}\left(h_{1}\right)(F) \mid h_{2}\left(h_{1}(G)\right)\right)_{x}$, then, by the bounded distortion condition with respect to $h_{1}(G)$, we obtain that $\left(h_{2}\left(h_{1}\right)(F) \mid h_{2}\left(h_{1}(G)\right)\right)_{x}-$ $\left(h_{2}\left(h_{1}\right)(F) \mid h_{2}\left(h_{1}(H)\right)\right)_{x} \leq \ln \left(\mathcal{K}_{2}\right)$, proving the claim. Hence, we will assume that $\left(h_{2}\left(h_{1}\right)(F) \mid h_{2}\left(h_{1}(H)\right)\right)_{x} \geq\left(h_{2}\left(h_{1}\right)(F) \mid h_{2}\left(h_{1}(G)\right)\right)_{x}$.

Let $\mathcal{F}_{i}$ be the set of end points $F^{\prime} \in \operatorname{end}\left(T^{\prime}, w\right)$ such that $\left(h(F) \mid F^{\prime}\right)_{w}=t_{0}+i$ with $i=0, t_{1}-t_{0}$. For any $F_{1}^{\prime}, F_{2}^{\prime} \in \mathcal{F}_{i}$, the bounded distortion condition with respect to $h_{1}(F)$ implies that

$$
\left|\left(h_{2}\left(h_{1}(F)\right) \mid h_{2}\left(F_{1}^{\prime}\right)\right)_{x}-\left(h_{2}\left(h_{1}(F)\right) \mid h_{2}\left(F_{2}^{\prime}\right)\right)_{x}\right| \leq \ln \left(\mathcal{K}_{2}\right) .
$$

Thus, if

(3) $\left(h_{2}\left(h_{1}\right)(F) \mid h_{2}\left(h_{1}(H)\right)\right)_{x}-\left(h_{2}\left(h_{1}\right)(F) \mid h_{2}\left(h_{1}(G)\right)\right)_{x}>\left(t_{1}-t_{0}+1\right) \ln \left(\mathcal{K}_{2}\right)+C$,

since the vertices in $T^{\prime \prime}$ have valence at least 3 , then there are $C$ end points $F_{j}^{\prime \prime}$, $j=1, C$, in $\operatorname{end}\left(T^{\prime \prime}, x\right)$ such that $\left(h_{2}\left(h_{1}(F)\right) \mid h_{2}\left(h_{1}(G)\right)_{x}<\left(h_{2}\left(h_{1}(F)\right) \mid F_{j}^{\prime \prime}\right)_{x}<\right.$ $\left(h_{2}\left(h_{1}(F)\right) \mid h_{2}\left(h_{1}(H)\right)_{x}\right.$ which are not in $h_{2}\left(\mathcal{F}_{i}\right)$ for any $i=0, t_{1}-t_{0}$ and such that $\left(h_{2}\left(h_{1}(F)\right) \mid F_{j_{1}}^{\prime \prime}\right)_{x} \neq\left(h_{2}\left(h_{1}(F)\right) \mid F_{j_{2}}^{\prime \prime}\right)_{x}$ for any $j_{1} \neq j_{2}$. Suppose $C=\ln \left(\mathcal{K}_{2}\right)+1$.

Therefore, either $\left(h_{2}\left(h_{1}\right)(F) \mid h_{2}\left(h_{1}(H)\right)\right)_{x}-\left(h_{2}\left(h_{1}\right)(F) \mid h_{2}\left(h_{1}(G)\right)\right)_{x} \leq\left(t_{1}-t_{0}+\right.$ 1) $\ln \left(\mathcal{K}_{2}\right)+C$ or there is some $F_{j_{0}}^{\prime \prime}$ so that $\left(h_{2}\left(h_{1}\right)(F) \mid h_{2}\left(h_{1}(H)\right)\right)_{x}-\left(h_{2}\left(h_{1}\right)(F) \mid F_{j_{0}}^{\prime \prime}\right)_{x}$ $\geq C$ and $\left(h_{2}\left(h_{1}\right)(F) \mid F_{j_{0}}^{\prime \prime}\right)_{x}-\left(h_{2}\left(h_{1}\right)(F) \mid h_{2}\left(h_{1}(G)\right)\right)_{x} \geq C$ with $h_{2}^{-1}\left(F_{j_{0}}^{\prime \prime}\right) \notin \mathcal{F}_{i}$ for 
any $i$, which means that either $\left(h_{1}(F), h_{2}^{-1}\left(F_{j_{0}}^{\prime \prime}\right)\right)_{w}<t_{0}$ or $\left(h_{1}(F), h_{2}^{-1}\left(F_{j_{0}}^{\prime \prime}\right)\right)_{w}>t_{1}$. In both cases, the bounded distortion condition does not hold and (3) leads to a contradiction.

From this we conclude that $\left(h_{2}\left(h_{1}\right)(F) \mid h_{2}\left(h_{1}(H)\right)\right)_{x}-\left(h_{2}\left(h_{1}\right)(F) \mid h_{2}\left(h_{1}(G)\right)\right)_{x} \leq$ $\left(t_{1}-t_{0}+1\right) \ln \left(\mathcal{K}_{2}\right)+\ln \left(\mathcal{K}_{2}\right)+1 \leq\left(\ln \left(\mathcal{K}_{1}\right)+1\right) \ln \left(\mathcal{K}_{2}\right)+\ln \left(\mathcal{K}_{2}\right)+1$ proving the claim.

Thus,

$$
\ln \left(\mathcal{K}_{3}\right)=\ln \left(\mathcal{K}_{(T, v),\left(T^{\prime \prime}, x\right)}\right) \leq \ln \left(\mathcal{K}_{1}\right) \cdot \ln \left(\mathcal{K}_{2}\right)+2 \ln \left(\mathcal{K}_{2}\right)+1
$$

Since $\varrho\left((T, v),\left(T^{\prime \prime}, x\right)\right)=\ln \left(1+2 \ln \left(\mathcal{K}_{3}\right)\right)$ it follows that

$$
\varrho\left((T, v),\left(T^{\prime \prime}, x\right)\right) \leq \ln \left(1+2 \ln \left(\mathcal{K}_{1}\right) \cdot \ln \left(\mathcal{K}_{2}\right)+4 \ln \left(\mathcal{K}_{2}\right)+2\right) .
$$

Now, observe that $\varrho\left((T, v),\left(T^{\prime}, w\right)\right)+\varrho\left(\left(T^{\prime}, w\right),\left(T^{\prime \prime}, x\right)\right)=\ln \left(1+2 \ln \left(\mathcal{K}_{1}\right)\right)+$ $\ln \left(1+2 \ln \left(\mathcal{K}_{2}\right)\right)=\ln \left(4 \ln \left(\mathcal{K}_{1}\right) \cdot \ln \left(\mathcal{K}_{2}\right)+2 \ln \left(\mathcal{K}_{1}\right)+2 \ln \left(\mathcal{K}_{2}\right)+1\right)$.

Since $\ln \left(\mathcal{K}_{1}\right) \geq 1$, from (4) we obtain that

$$
\begin{gathered}
\varrho\left((T, v),\left(T^{\prime \prime}, x\right)\right) \leq \ln \left(1+2 \ln \left(\mathcal{K}_{1}\right) \cdot \ln \left(\mathcal{K}_{2}\right)+2 \ln \left(\mathcal{K}_{1}\right) \cdot \ln \left(\mathcal{K}_{2}\right)+2 \ln \left(\mathcal{K}_{2}\right)+2 \ln \left(\mathcal{K}_{1}\right)\right) \\
=\varrho\left((T, v),\left(T^{\prime}, w\right)\right)+\varrho\left(\left(T^{\prime}, w\right),\left(T^{\prime \prime}, x\right)\right) .
\end{gathered}
$$

Remark 4.9. Given $(T, v),\left(T^{\prime}, w\right) \in[(R, z)], \varrho\left((T, v),\left(T^{\prime}, w\right)\right) \in\{\ln (1+2 n) \mid n=$ $1,2 \ldots\}$.

Remark 4.10. $([(R, z)], \varrho)$ is discrete.

Remark 4.11. As Mosher, Sageev, and Whyte [13, page 118] point out, any two bounded valence (i.e. there is a constant $A$ such that the valence of every vertex is less than $A$ ), locally finite, simplicial, bushy trees are quasi-isometric. Therefore, any such tree is quasi-isometric to the infinite binary tree. See also Bridson and Haefliger [3, page 141, Exercise 8.20(2)] for the special case of regular, simplicial trees.

Let $\mathcal{T}_{\geq 3}^{*}$ be the class of bounded valence trees in $\mathcal{T}_{\geq 3}$. From Remark 4.11 it follows that

Proposition 4.12. $\left(\mathcal{T}_{\geq 3}^{*}, \varrho\right)$ is a metric space.

Given a rooted simplicial tree $(T, v)$ and a vertex $x \in T$, let us denote the order of $x$ by $\operatorname{ord}(x)$ and let us call the vertices whose geodesic to the root $v$ contains $x$, descendants of $x$. If $k \in \mathbb{N}$, by $\operatorname{desc}_{k}(x)$ we denote the set of descendants of $x$ at a distance $k$ with the canonical metric on the tree (edges having length 1 ).

Given $x \in(T, v)$, we denote by $T_{x}=\{y \in T \mid x \in[v, y]\}$, which is also a tree. If $(T, v)$ is geodesically complete, so is $\left(T_{x}, x\right)$ and there is a canonical injection $j: \operatorname{end}\left(T_{x}, x\right) \rightarrow \operatorname{end}(T, v)$. Abusing the notation, we may consider $\operatorname{end}\left(T_{x}, x\right) \subset$ $\operatorname{end}(T, v)$.

Lemma 4.13. Let $(T, v),\left(T^{\prime}, w\right)$ be two rooted simplicial trees and $x$ be a vertex in $T$. If $\operatorname{ord}(x)=m+1$ and $\mathcal{D}=\min _{k}\left\{\sup _{x^{\prime} \in \mathcal{V}\left(T^{\prime}\right)}\left\{\operatorname{desc}_{k}\left(x^{\prime}\right)\right\}<m\right\}$, then $\varrho\left((T, v),\left(T^{\prime} w\right)\right)>\ln (1+2 \mathcal{D})$.

Proof. Consider any bijection $h: \operatorname{end}(T, v) \rightarrow \operatorname{end}\left(T^{\prime} w\right)$. Let $x_{1}, \ldots, x_{m}$ be $m$ different vertices in $\operatorname{desc}_{1}(x)$ and let $F_{i} \in \operatorname{end}\left(T_{x_{i}}, x_{i}\right) \subset \operatorname{end}(T, v)$ for $i=1, m$. There is a unique vertex $y \in T^{\prime}$ such that $h\left(F_{i}\right) \in \operatorname{end}\left(T_{y}^{\prime}, y\right) \forall i=1, m$ and 
$|w y|$ is maximal. Then, since the cardinal of $\operatorname{desc}_{\mathcal{D}}(y)$ is less than $m$, there are $F_{i_{1}}, F_{i_{2}}, F_{i_{3}}, i_{j} \in\{1, m\}$ such that $\left[h\left(F_{i_{1}}\right) \mid h\left(F_{i_{2}}\right)\right]=[w, y],\left[h\left(F_{i_{1}}\right) \mid h\left(F_{i_{3}}\right)\right]=[w, y]$ and $\left(h\left(F_{i_{2}}\right) \mid h\left(F_{i_{3}}\right)\right)_{w}>|w y|+\mathcal{D}$. Hence, $D_{h}\left(F_{1}, e^{-\|x\|}\right)>\mathcal{D}$ and $\varrho\left((T, v),\left(T^{\prime} w\right)\right)>$ $\ln (1+2 \mathcal{D})$.

Proposition 4.14. $\left(\mathcal{T}_{\geq 3}^{*}, \varrho\right)$ is unbounded.

Proof. Consider $T_{m}$ and $T_{3}$ to be the trees in $\mathcal{T}_{>3}^{*}$ where every vertex has order $m$ and 3 respectively. As we said in Remark 4.11 $T_{m}$ and $T_{3}$ are in the same quasi-isometry class. Therefore, it is trivial to check that

$$
\lim _{m \rightarrow \infty} \min _{k}\left\{\sup _{x^{\prime} \in \mathcal{V}\left(T_{3}\right)}\left\{\operatorname{desc}_{k}\left(x^{\prime}\right)\right\}<m\right\}
$$

and, by Lemma 4.13. $\left(\mathcal{T}_{\geq 3}^{*}, \varrho\right)$ is unbounded.

The function $\varrho$ can also be used to define a metric between some classes of trees in $\mathcal{T}$.

Let us define $(T, v) \sim\left(T^{\prime}, w\right)$ if they have the same branching. Then $\sim$ is an equivalence relation in $\mathcal{T}$. Moreover, for any $(T, v) \in \mathcal{T}$ there is a unique tree $\left(T^{\prime}, w\right) \in \mathcal{T}_{\geq} 3$ such that $(T, v) \sim\left(T^{\prime}, w\right)$. Let us denote by $[T, v]$ the equivalence class of $(T, v)$ and by $[T, v]_{\geq 3}$ the unique representative of the class in $\mathcal{T}_{\geq} 3$.

Now, for any $(T, v),\left(T^{\prime}, w\right) \in \mathcal{T}$, if $[T, v]_{\geq 3}$ and $\left[T^{\prime}, w\right]_{\geq 3}$ are quasi-isometric we can define

$$
\sigma\left([T, v],\left[T^{\prime}, w\right]\right):=\varrho\left([T, v]_{\geq 3},\left[T^{\prime}, w\right]_{\geq 3}\right) .
$$

Let $\mathcal{T}^{*}$ be the category of bounded valence, locally finite, simplicial, bushy trees. This, together with Proposition 4.12 and Remark 4.11, yields

Proposition 4.15. $\left(\mathcal{T}^{*} / \sim, \sigma\right)$ is an unbounded metric space.

\section{REFERENCES}

[1] L. V. Ahlfors, Lectures on Quasiconformal Mappings. Second edition. With supplemental chapters by C. J. Earle, I. Kra, M. Shishikura and J. H. Hubbard. University Lecture Series, 38. American Mathematical Society, Providence, RI, 2006. MR.2241787 (2009d:30001)

[2] M. Bonk and O. Schramm, Embeddings of Gromov hyperbolic spaces. Geom. Funct. Anal. 10, No. 2 (2000), 266-306. MR1771428 (2001g:53077)

[3] M. Bridson and A. Haefliger, Metric spaces of non-positive curvature. Springer-Verlag, Berlin, 1999. MR 1744486 (2000k:53038)

[4] S. Buyalo and V. Schroeder, Elements of Asymptotic Geometry. EMS Monographs in Mathematics. EMS Publishing House, Zürich, 2007. MR2327160 (2009a:53068)

[5] V. Z. Fel̆nberg, Compact ultrametric spaces. Dokl. Akad. Nauk SSSR 214 (1974), 1041-1044. MR0348715 (50:1212)

[6] É. Ghys and P. de la Harpe, Sur les groupes hyperboliques d'après Mikhael Gromov Progr. Math. 83, Birkhäuser, Boston, MA, 1990. MR1086648 (92f:53050)

[7] J. Heinonen, Lectures on analysis on metric spaces, Universitext, Springer-Verlag, New York, 2001. MR1800917 (2002c:30028)

[8] J. H. Hubbard, Teichmüller Theory and Applications to Geometry, Topology, and Dynamics. (Volume I: Teichmüller Theory). Matrix Editions, Ithaca, NY, 2006. MR2245223 (2008k:30055)

[9] B. Hughes, Trees and ultrametric spaces: a categorical equivalence. Adv. Math. 189 (2004), 148-191. MR2093482(2005m:57001)

[10] B. Hughes, A. Martínez-Pérez and M. A. Morón, Bounded distortion homeomorphisms on ultrametric spaces. Ann. Acad. Sci. Fenn. Math. 35 (2010), 473-492. MR2731702

[11] Á. Martínez-Pérez and M. A. Morón, Uniformly continuous maps between ends of $\mathbb{R}$-trees. Math. Z. 263, No. 3 (2009), 583-606. MR2545858(2010j:54049) 
[12] Á. Martínez-Pérez, Quasi-isometries between visual hyperbolic spaces. Manuscripta Math. (2011), DOI 10.1007/s00229-011-0463-8.

[13] L. Mosher, M. Sageev and K. Whyte, Quasi-actions on trees I. Bounded valence. Ann. of Math. (2) 158 (2003), 115-164. MR1998479 (2004h:20055)

[14] F. Paulin, Un groupe hyperbolique est déterminé par son bord. J. Lond. Math. Soc. (2) 54 (1996), 50-74. MR1395067 (97d:20042)

Departamento de Geometría y Topología, Universidad Complutense de Madrid, MADRID 28040, SPAIN

E-mail address: alvaro_martinez@mat.ucm.es 